\title{
Managing a group of qualified veterinary technicians
}

Great Eastern University was justifiably proud of its veterinary technicians. All were certified Laboratory Animal Technicians or Technologists, some were certified by the Academy of Surgical Research, and all were well-trained and competent in the tasks assigned to them. At Great Eastern, the veterinary technicians worked closely with the veterinarians to provide health care to the school's animals and, on a fee-forservice basis, provided technical assistance for investigators, particularly during surgical procedures.

Dr. Patty Hagen usually had veterinary technician Pam Cronin assisting her. Cronin and a second technician would anesthetize Hagen's pigs and prepare them for surgery. Cronin then would serve as the anesthetist during surgery and would also provide any immediate postoperative care. If Cronin was not available, her supervisor would assign a different but equally competent technician to work with Hagen. Hagen, the IACUC and the veterinary staff were all happy with this arrangement. However, one person wasn't. During a routine visit to Great Eastern, the USDA veterinary inspector reviewed Hagen's protocol and noticed in the file a separate form documenting the drugs used and vital signs monitored while the pig was under anesthesia. Cronin had signed the anesthesia monitoring form but was not listed on the approved IACUC protocol. A few questions revealed that Hagen did not know in advance which technician would be helping her during surgery, and so she could not list a specific person on the IACUC protocol form. Rather, the IACUC had a written policy stating that the vivarium's veterinary technician supervisor was empowered to assign any veterinary technician to work with any investigator, as long as the technician had the appropriate training and experience for the task at hand. The supervisor kept a regularly updated list of each technician's skills and training. Nevertheless, the school was issued a citation for noncompliance with $\$ 2.32$.a and $\$ 2.32$.b of the Animal Welfare Act regulations ${ }^{1}$, stating that the IACUC did not provide adequate review of personnel qualifications, instead allowing the technician supervisor to make that determination. For its part, the school considered appealing the citation, because the committee had voted affirmatively for the existing policy and the technician supervisor was acting on behalf of the IACUC.

Do you think that the IACUC or the USDA inspector was right in this case? What recommendations would you make to prevent a recurrence of this problem?

1. Animal Welfare Act and Regulations. 9 CFR, Chapter 1, Subchapter A.

\section{RESPONSE}

\section{Close the loop}

\section{Kimberly S. Edgar, MBA}

The old saying, "if something is not documented, it didn't happen," applies to this case. A lack of documentation explains why Great Eastern received a citation from the USDA for noncompliance with regulations governing personnel training and qualifications.

The university's veterinary technical staff members have achieved Laboratory Animal Technician or Technologist certifications, and some of the technicians have obtained rigorous and specialized certification by the Academy of Surgical Research. The veterinary technician supervisor appears to be an effective supervisor; he or she has fostered a team of competent veterinary technicians, provided support to obtain additional professional certifications and provided cross-training opportunities. This achieves many objectives of a veterinary care program and ensures that the veterinary technical staff can effectively cover all aspects of the program's services, including technical assistance for pig surgery.

Because the technicians are qualified for this work, the problem lies in a lack of documentation of their qualifications. I believe Great Eastern should appeal the citation. But before doing so, it should formulate a long-term strategy to document personnel training and qualifications. This strategy should include revising the IACUC protocol form to include statements that the qualified veterinary technical staff provides pre-surgical, surgical and post-surgical care on research surgical protocols when requested by the principal investigator. The strategy should also include maintaining a database of veterinary technical staff members and their training, qualifications and assessments, including species-specific and procedure-specific skills. This database should be reviewed by the Institutional Official, the IACUC administrative staff and the IACUC during their semi-annual program reviews. The IACUC should approve the veterinary technicians' qualifications as part of its evaluation process of the veterinary care program. This IACUC policy should cover the approval of the veterinary technical staff providing anesthesia, surgical and post-operative care on IACUC-approved surgical research protocols. Great Eastern should also develop a standard operating procedure to cover the veterinary technical staff assigned to specific surgical cases by providing consistent documentation on the anesthesia monitoring form and generating a paper trail on each case. In addition, the veterinary technicians conducting post-approval monitoring on the pigs 